\title{
Inclusive and exclusive processes with a leading neutron in ep collisions
}

\author{
V. P. Goncalves* \\ Department of Astronomy and Theoretical Physics, Lund University, Lund, Sweden \\ High and Medium Energy Group, Instituto de Física e Matemática, Universidade Federal de \\ Pelotas, Pelotas, Brasil \\ E-mail: barros@ufpel .edu.br

\section{F. Carvalho} \\ Departamento de Ciências Exatas e da Terra, Universidade Federal de São Paulo, \\ Campus Diadema, Diadema, SP, Brazil
}

\section{F. S. Navarra}

Instituto de Física, Universidade de São Paulo, São Paulo, SP, Brazil

\section{Spiering}

Instituto de Física, Universidade de São Paulo, São Paulo, SP, Brazil

\begin{abstract}
The inclusive and exclusive processes associated with a leading neutron in $e p$ collisions at high energies are described using the color dipole formalism. We demonstrate that the available experimental HERA data on the $x_{L}$ (Feynman momentum) distribution of leading neutrons can be very well described taking into account the nonlinear (saturation) effects in the QCD dynamics. Moreover, we present our estimate for the exclusive cross sections which can be studied at HERA and in future electron-proton colliders.
\end{abstract}

XXIV International Workshop on Deep-Inelastic Scattering and Related Subjects

11-15 April, 2016

DESY Hamburg, Germany

\footnotetext{
* Speaker.
} 


\section{Introduction}

HERA has provided high precision experimental data on semi - inclusive $e+p \rightarrow e+n+X$ processes, where the incident proton is converted into a neutron via a charge exchange [1]. Very recently the first measurements of exclusive $\rho$ photoproduction associated with leading neutrons $\left(\gamma p \rightarrow \rho^{0} \pi^{+} n\right)$ were presented [2]. The description of these leading neutron processes is still a theoretical challenge. In particular, the $x_{L}$ (Feynman momentum) distribution of leading neutrons remains without a conclusive theoretical description. In Refs. [3, 4] we proposed an unified description of inclusive and exclusive processes with a leading neutron, based on the color dipole formalism, and we have demonstrated that the available experimental HERA data on the $x_{L}$ (Feynman momentum) distribution of leading neutrons can be very well described in this approach. An important property of our approach is that its main elements are constrained by the HERA data on processes without a leading neutron. As a consequence, our analysis of leading neutron data has put limits on the magnitude of the nonperturbative absorptive corrections and on the models of the pion flux (which describes the pion emission by the incident proton). Moreover, we were able to present parameter - free predictions for the inclusive and exclusive processes with a leading neutron at the energies of the future $e p$ colliders [5]. In what follows we will review the basic aspects of the formalism in Section 2 and present our main results in Section 3. For more details we refer the reader to the Refs. [3, 4].

\section{Formalism}

At high energies, the differential cross section for a given process (semi - inclusive or exclusive) associated with a leading neutron production can be expressed as follows:

$$
\frac{d^{2} \sigma\left(W, Q^{2}, x_{L}, t\right)}{d x_{L} d t}=f_{\pi / p}\left(x_{L}, t\right) \sigma_{\gamma^{*} \pi}\left(\hat{W}^{2}, Q^{2}\right)
$$

where $Q^{2}$ is the virtuality of the exchanged photon, $W$ is the center-of-mass energy of the virtual photon-proton system, $x_{L}$ is the proton momentum fraction carried by the neutron and $t$ is the square of the four-momentum of the exchanged pion. Moreover, $f_{\pi / p}$ is the flux of virtual pions emitted by the proton and $\sigma_{\gamma^{*} \pi}\left(\hat{W}^{2}, Q^{2}\right)$ is the cross section of the interaction between the virtual-photon and the virtual-pion at center-of-mass energy $\hat{W}$, which is given by $\hat{W}^{2}=\left(1-x_{L}\right) W^{2}$. The pion flux $f_{\pi / p}\left(x_{L}, t\right)$ (also called sometimes pion splitting function) is the virtual pion momentum distribution in a physical nucleon (the bare nucleon plus the "pion cloud"). In Ref. [3], we described the semi - inclusive leading neutron processes in the color dipole formalism. The basic idea is that at high energies, this process can be seen as a sequence of three factorizable subprocesses [See Fig. 1 (left panel)]: i) the photon fluctuates into a quark-antiquark pair (the color dipole), ii) the color dipole interacts with the pion, present in the wave function of the incident proton, and iii) the leading neutron is formed. Consequently, the photon - pion cross section can be factorized in terms of the photon wave functions $\Psi$, which describes the photon splitting in a $q \bar{q}$ pair, and the dipole-pion cross section $\sigma_{d \pi}$. We have

$$
\sigma_{\gamma^{*} \pi}\left(\hat{x}, Q^{2}\right)=\int_{0}^{1} d z \int d^{2} r \sum_{L, T}\left|\Psi_{T, L}\left(z, r, Q^{2}\right)\right|^{2} \sigma_{d \pi}(\hat{x}, r)
$$



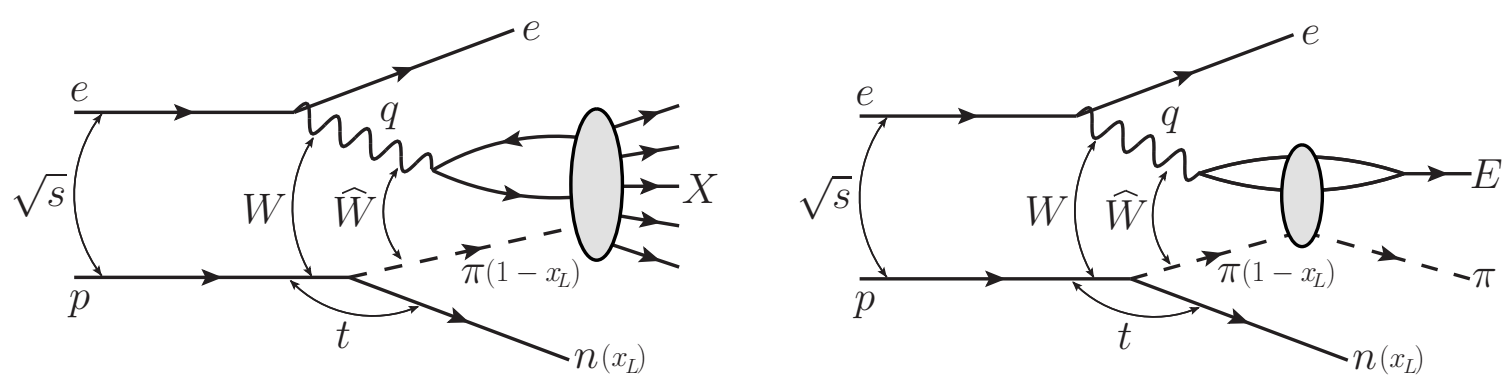

Figure 1: Semi - inclusive (left panel) and exclusive (right panel) ep processes associated with a leading neutron $n$ production in the color dipole formalism.

where in the eikonal approximation the dipole-proton cross section $\sigma_{d \pi}$ is given by:

$$
\sigma_{d \pi}(\hat{x}, r)=2 \int d^{2} b \mathscr{N}^{\pi}(\hat{x}, r, b)
$$

with

$$
\hat{x}=\frac{Q^{2}+m_{f}^{2}}{\hat{W}^{2}+Q^{2}}=\frac{Q^{2}+m_{f}^{2}}{\left(1-x_{L}\right) W^{2}+Q^{2}}
$$

being the scaled Bjorken variable and $\mathscr{N}^{\pi}(x, r, b)$ is the imaginary part of the forward amplitude of the scattering between a small dipole (a colorless quark-antiquark pair) and a pion, at a given rapidity interval $Y=\ln (1 / \hat{x})$. In Ref. [3] we proposed to relate $\mathscr{N}^{\pi}$ with the dipole-proton scattering amplitude $\mathscr{N}^{p}$, usually probed in the typical inclusive and exclusive processes at HERA, assuming that

$$
\mathscr{N}^{\pi}(\hat{x}, r, b)=R_{q} \cdot \mathscr{N}^{p}(\hat{x}, r, b)
$$

with $R_{q}$ being a constant. Since the effective value of this quantity is still an open question, we have considered in [3] that $R_{q}$ could be in the range $1 / 3 \leq R_{q} \leq 2 / 3$.

As mentioned in Ref. [3], one source of uncertainty in the study of inclusive leading neutron production (in Fig. 1 on the left) is the fact that there are several processes which lead to the same final state. Apart from one pion emission we may have, for example, $\rho$ emission. Even with pion emission we may have $\Delta$ production with the subsequent decay $\Delta \rightarrow n+\pi$. The strength of these contributions is highly model dependent and their existence prevents us from extracting more precise information on the photon-pion cross section or on the pion flux. In contrast, in $\rho$ exclusive production with a leading neutron none of these processes contributes to the exclusive reaction shown in the right panel of Fig. 1. This feature makes the leading neutron spectrum measured in exclusive processes a better testing ground for both the determination of the photon-pion cross section and of the pion flux. In Ref. [4] we assumed that the factorization given by Eq. (2.1) also is valid and that the photon - pion cross section for the production of an exclusive final state $E$, such as a vector meson $(E=V)$ or a real photon in DVCS $(E=\gamma)$, in the $\gamma^{*} \pi \rightarrow E \pi$ process is given in the color dipole formalism by:

$$
\sigma\left(\gamma^{*} \pi \rightarrow E \pi\right)=\sum_{i=L, T} \int_{-\infty}^{0} \frac{d \sigma_{i}}{d \hat{t}} d \hat{t}=\frac{1}{16 \pi} \sum_{i=L, T} \int_{-\infty}^{0}\left|\mathscr{A}_{i}^{\gamma^{*} \pi \rightarrow E \pi}(x, \Delta)\right|^{2} d \hat{t}
$$




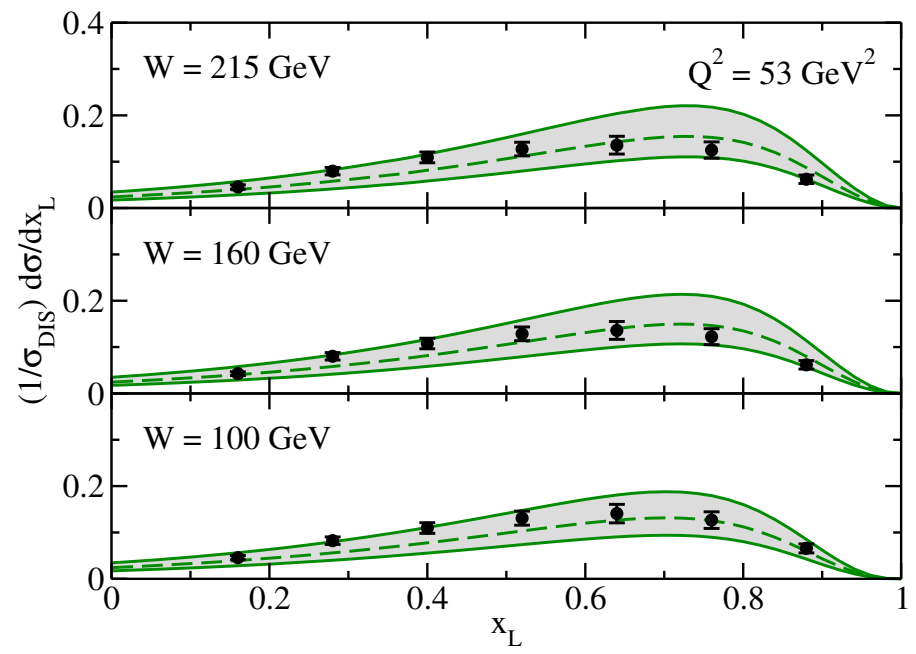

Figure 2: Leading neutron $x_{L}$ spectrum for three photon-proton energies compared with recent H1 data.

with the scattering amplitude being given by

$$
\mathscr{A}_{T, L}^{\gamma^{*} \pi \rightarrow E \pi}(\hat{x}, \Delta)=i \int d z d^{2} r d^{2} b e^{-i[b-(1-z) r] \cdot \Delta}\left(\Psi^{E *} \Psi\right)_{T, L} 2 \mathscr{N}_{\pi}(\hat{x}, r, b)
$$

where $\left(\Psi^{E *} \Psi\right)_{T, L}$ denotes the overlap of the photon and exclusive final state wave functions. The variable $z(1-z)$ is the longitudinal momentum fractions of the quark (antiquark) and $\Delta$ denotes the transverse momentum lost by the outgoing pion $\left(\hat{t}=-\Delta^{2}\right)$. The variable $b$ is the transverse distance from the center of the target to the center of mass of the $q \bar{q}$ dipole and the factor in the exponential arises when one takes into account non-forward corrections to the wave functions. In our study we have assumed that the vector meson is predominantly a quark-antiquark state and that the spin and polarization structure is the same as in the photon and can be described by the Boosted Gaussian and Gauss-LC models described in Ref. [4]. Finally, in order to estimate the photon - pion cross section we must specify the dipole - pion scattering amplitude $\mathscr{N}^{\pi}$. In Refs. $[3,4]$ we assumed the validity of the approximation expressed by Eq. (2.5), with the dipole proton scattering amplitude $\mathscr{N}^{p}$ being given by the bCGC model, which is based on the CGC formalism and takes into account the impact parameter dependence of the dipole - proton scattering amplitude. Additionally, we have considered that absorptive corrections, associated to extra soft interactions, only modify the normalization of the dipole - pion scattering amplitude given in Eq. (2.5) and can be modelled by a constant (independent of $x_{L}$ ) factor $K$, which is fixed by the comparison between our predictions and the experimental data.

\section{Results}

In Fig. 2 we compare our predictions for the inclusive leading neutron production with the experimental data. All the parameters contained in the dipole cross section have been already fixed by the analysis of other DIS data from HERA. The pion flux has been fixed by fitting data of low energy hadronic collisions. The bands account for the uncertainty in the choice of the factors $R_{q}$ 

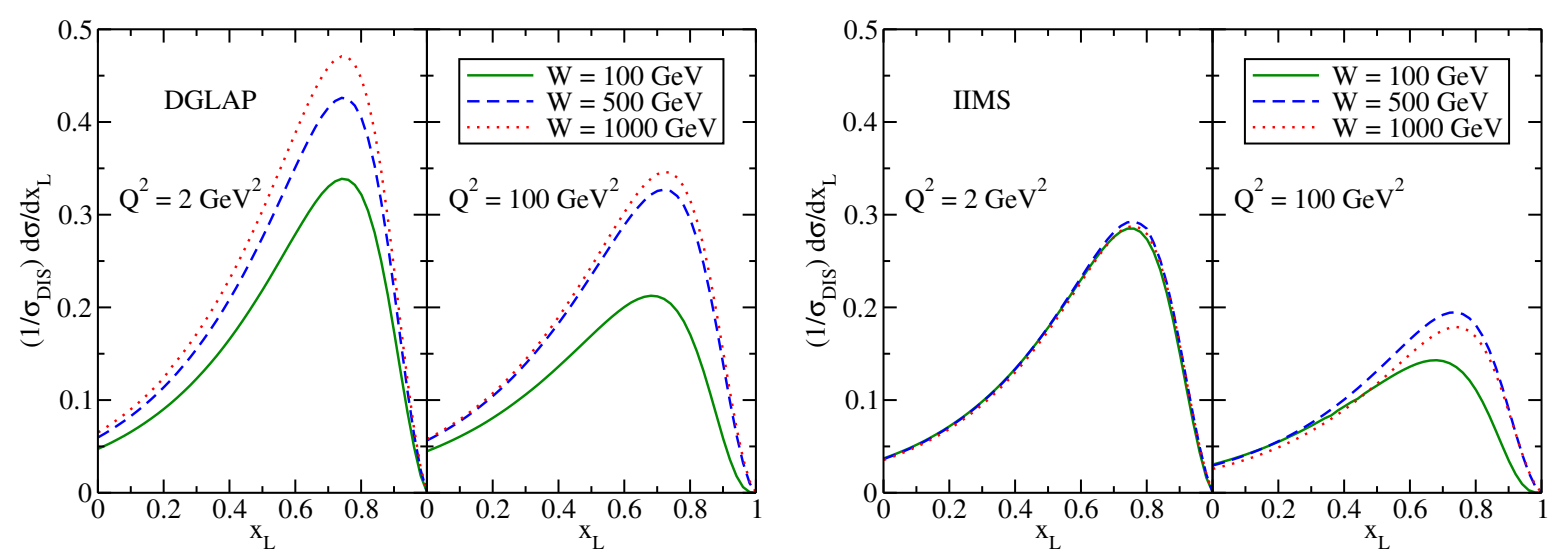

Figure 3: Leading neutron spectra for different energies considering the linear DGLAP model (left panel) and the nonlinear saturation model (right panel) for the dipole-pion cross section.

and $K$. The upper limit of each band corresponds to the choice $R_{q}=2 / 3$ and $K=1$ whereas the lower limit corresponds to the choice $R_{q}=0.5$ and $K=0.5$. The central dashed lines represent the choice where $R_{q} \cdot K=0.5$, which could, for example, be realized with $R_{q}=0.5$ and $K=1$.

In Ref. [3] we analyse the Feynman scaling in the leading neutron spectra and the contribution from nonlinear effects to this process. As demonstrated in [3] this process is surprisingly sensitive to low $x$ physics. In particular, the large $x_{L}\left(x_{L}>0.3\right)$ we observe the transition from the large to small $x$ domain. It is therefore interesting to check whether gluon saturation effects are already playing a significant role. The energy dependence is contained in the dipole forward scattering amplitude $\mathscr{N}$. Therefore, we can estimate the contribution of the nonlinear effects comparing the results obtained with saturation model with those obtained with the linear model. Moreover, the theoretical expectation can be obtained using the GBW model for the scattering amplitude, where $\mathscr{N}=1-\exp \left(-r^{2} Q_{s}^{2}(\hat{x}) / 4\right)$. In the linear limit, when the dipole radius is very small (or equivalently $Q^{2}$ is very large) or the saturation scale is very small (and hence the energy is not very high), we obtain

$$
\sigma_{d \pi}(r, \hat{x}) \propto \sigma_{0} \mathscr{N}(r, \hat{x}) \simeq \sigma_{0} \frac{Q_{s}^{2}(\hat{x}) r^{2}}{4} \simeq \sigma_{0} Q_{0}^{2} x_{0}^{\lambda}\left[\frac{\left(1-x_{L}\right) W^{2}+Q^{2}}{Q^{2}+m_{f}^{2}}\right]^{\lambda} .
$$

Consequently, in this regime we see that the leading neutron $x_{L}$ spectrum will depend on $W$. In a complementary way, in the nonlinear limit, when the dipole radius is very large (or equivalently $Q^{2}$ is very small) or the saturation scale is very large (and hence the energy is very high), we obtain

$$
\sigma_{d \pi}(r, \hat{x}) \propto \sigma_{0} \mathscr{N}(r, \hat{x}) \simeq \sigma_{0}
$$

which is energy independent. These expectations can be compared with those obtained using the IIMS and DGLAP models for the dipole-pion cross section. In Fig. 3 (a) we show the spectra obtained in a purely linear approach. As expected we see a noticeable energy dependence. In contrast, the non linear predictions presented in Fig. 3 (b) show a remarkable suppression of the energy dependence at low values of $Q^{2}$, consistent with the expectations. These results indicate that the Feynman scaling (and how it is violated) can be directly related to the QCD dynamics at small- $x$. 


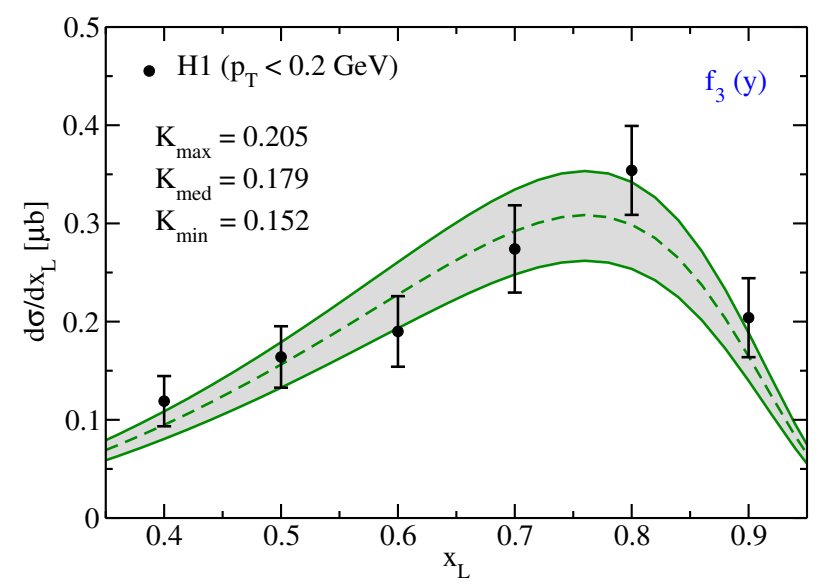

Figure 4: Leading neutron spectra in exclusive $\rho$ photoproduction obtained considering the possible range of values of the $K$ factor. Data from Ref. [2].

Let us start our analysis of the exclusive processes considering the exclusive $\rho$ photoproduction associated with leading neutrons as analysed by the H1 Collaboration [2]. We have assumed that $W=60 \mathrm{GeV}, Q^{2}=0.04 \mathrm{GeV}^{2}$ and that $p_{T}<0.2 \mathrm{GeV}$, where $p_{T}$ is the transverse momentum of the leading neutron. Moreover, we will assume initially that $R_{q}=2 / 3$. In Ref. [4] we have considered different models for the dipole - proton scattering amplitude and pion flux and estimated the dependence of our results on these choices. Moreover, in order to constrain the value of the $K$ factor associated to absorptive corrections, we have assumed the following strategy: for a given model of the pion flux, $R_{q}$ and dipole cross section, we estimated the total cross section. The value of $K$ was the value necessary to make our prediction consistent with the H1 data [2]. We have obtained a reasonable agreement with the experimental data, with $K$ values in the range $0.134<K<0.210$. It is important to emphasize that these values of $K$ are strongly correlated with our choice for $R_{q}$. For example, if instead of $R_{q}=2 / 3$ we assume $R_{q}=1 / 3$, the corresponding $K$ values should be multiplied by 4 , since the exclusive cross sections depend quadratically on the dipole scattering amplitude. In Ref. [4] we find that the different predictions for the $x_{L}$ spectra are very similar. However, the effective value of the absorptive correction $K$ depends on the model of the dipole scattering amplitude as expected. In Fig. 4 we present our predictions taking into account the experimental uncertainty present in the $\mathrm{H} 1$ data for the total cross section [2], which in our analysis translates into a range of possible values for the $K$ factor. These results indicate that the experimental data can be quite well described by the dipole approach.

Considering that the main inputs of our calculations have been fixed by the experimental data on exclusive $\rho$ photoproduction we can extend our analysis to other exclusive final states. We will assume the Gauss-LC model for vector meson wave function, the bCGC dipole scattering amplitude and the same the model for the pion flux and $K$ values needed to describe the $\rho$ data. Initially, let us consider the kinematical range probed by HERA. As in the $\rho$ case, we will assume $W=60 \mathrm{GeV}$ and $p_{T}<0.2 \mathrm{GeV}$. However, for $\phi$ and $J / \Psi$ production we assume $Q^{2}=0.04 \mathrm{GeV}^{2}$, while for the DVCS we consider that $Q^{2}=10 \mathrm{GeV}^{2}$. The corresponding predictions for the leading neutron spectra in exclusive $\phi$ and $J / \Psi$ production as well as in DVCS are presented in Fig. 5. For the HERA kinematical range we predict $\sigma(\gamma p \rightarrow \phi \pi n)=25.47 \pm 3.70 \mathrm{nb}, \sigma(\gamma p \rightarrow J / \Psi \pi n)=$ 

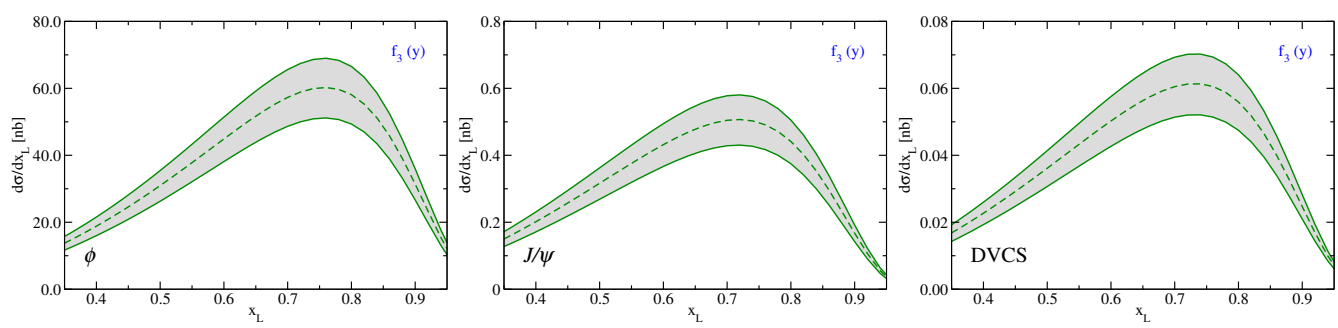

Figure 5: Predictions for the leading neutron spectra in exclusive $\phi, J / \Psi$ and DVCS production in the HERA kinematical range: $W=60 \mathrm{GeV}$ and $p_{T}<0.2 \mathrm{GeV}$.

$0.22 \pm 0.03 \mathrm{nb}$ and $\sigma\left(\gamma^{*} p \rightarrow \gamma \pi n\right)=0.008 \pm 0.001 \mathrm{nb}$, where the uncertainty is estimated taking into account the range of possible values of $K$. Finally, let us present our predictions for the kinematical range which may be probed in future ep colliders assuming $p_{T}<0.2 \mathrm{GeV}$. For $W=1$ $\mathrm{TeV}$ and $Q^{2}=5 \mathrm{GeV}^{2}$ we predict $\sigma\left(\gamma^{*} p \rightarrow \rho \pi n\right)=6.55 \pm 0.95 \mathrm{nb}, \sigma\left(\gamma^{*} p \rightarrow \phi \pi n\right)=1.71 \pm$ $0.25 \mathrm{nb}, \sigma\left(\gamma^{*} p \rightarrow J / \Psi \pi n\right)=1.20 \pm 0.17 \mathrm{nb}$ and $\sigma\left(\gamma^{*} p \rightarrow \gamma \pi n\right)=0.16 \pm 0.02 \mathrm{nb}$. We believe that for these values of total cross sections, the experimental analysis of the exclusive processes associated with a leading neutron is feasible in future $e p$ colliders. In particular, as the cross sections strongly increase when $Q^{2} \rightarrow 0$, the analysis of the vector meson photoproduction in $e p$ collisions can be useful to understand leading neutron spectra, which are of crucial importance in particle production in cosmic ray physics. Another possibility is the study of this process in ultraperipheral hadronic collisions, with the leading neutron being a tag for exclusive production. In principle these processes can be studied in the future at the LHC. Such proposition have been discussed in detail in the Ref. [6].

\section{References}

[1] V. Andreev et al. [H1 Collaboration], Eur. Phys. J. C 74, 2915 (2014); F. D. Aaron et al. [H1 Collaboration], Eur. Phys. J. C 68, 381 (2010).

[2] V. Andreev et al. [H1 Collaboration], Eur. Phys. J. C 76, 41 (2016).

[3] F. Carvalho, V. P. Goncalves, D. Spiering and F. S. Navarra, Phys. Lett. B 752, 76 (2016).

[4] V. P. Goncalves, F. S. Navarra and D. Spiering, Phys. Rev. D 93, 054025 (2016).

[5] D. Boer, M. Diehl, R. Milner, R. Venugopalan, W. Vogelsang, D. Kaplan, H. Montgomery and S. Vigdor et al., arXiv:1108.1713 [nucl-th].

[6] V. P. Goncalves, B. D. Moreira, F. S. Navarra and D. Spiering, Phys. Rev. D 94, 014009 (2016). 